\title{
Coexisting Kondo singlet state with antiferromagnetic long-range order: A possible ground state for Kondo insulators
}

\author{
Guang-Ming Zhang ${ }^{1}$ and $\mathrm{Lu} \mathrm{Yu}^{2,3}$ \\ 1 Center for Advanced Study, Tsinghua University, Beijing 100084, P. R. China \\ 2 International Center for Theoretical Physics, P. O. Box 586, Trieste 34100, Italy \\ ${ }^{3}$ Institute for Theoretical Physics, Academic Sinica, Beijing 100080, P. R. China
}

(November 3, 2018)

\begin{abstract}
The ground-state phase diagram of a half-filled anisotropic Kondo lattice model is calculated within a mean-field theory. For small transverse exchange coupling $J_{\perp}<J_{\perp c 1}$, the ground state shows an antiferromagnetic long-range order with finite staggered magnetizations of both localized spins and conduction electrons. When $J_{\perp}>J_{\perp c 2}$, the long-range order is destroyed and the system is in a disordered Kondo singlet state with a hybridization gap. Both ground states can describe the low-temperature phases of Kondo insulating compounds. Between these two distinct phases, there may be a coexistent regime as a result of the balance between local Kondo screening and magnetic interactions.

PACS numberes: 71.28.+d, 72.15.Qm, 75.20.Hr
\end{abstract}

The Kondo lattice model is often considered as a theoretical model for heavy fermion materials. For this model, an important issue arises from the interplay between the Kondo screening and the magnetic interactions among localized spins mediated by the conduction electrons. The former effect favors a nonmagnetic Kondo singlet state in strong coupling limit, while the latter interactions tend to stabilize a magnetically long-range ordered state in weak coupling limit. The nature of such a transition between these two distinct phases has been a long standing issue since it was first suggested by Doniach [1]. The one-dimensional model was intensively studied at half filling, showing that its ground state is always a disordered Kondo singlet state (or a spin liquid state) [2]. In higher dimensions, however, both antiferromagnetic long-range order (AFLRO) and disordered Kondo singlet states may occur [3]. Recently, there have been more indications of such a transition from various approximate treatments for the Kondo or Anderson lattice models, including variational Monte Carlo calculation [ $[$, higher-order series expansions [5], quantum Monte Carlo simulations [6,7], and infinite dimensional calculations [8].

In this paper, we would like to consider the issue whether the disordered Kondo singlet state can coexist with an AFLRO at half-filling. First of all, we introduce an anisotropic Kondo lattice model by distinguishing the longitudinal spin exchange interaction from the transverse one, because we notice that the longitudinal interaction describes a polarization of the conduction electrons by the localized spins, while the transverse one describes a spin-flip scattering of the conduction electrons off the localized spins. The former interaction is the origin of the magnetic interactions among localized spins, leading to an AFLRO; the latter interaction is responsible for the local Kondo screening effects, yielding a disordered Kondo singlet state. We also notice that the AFLRO and local Kondo singlet order operators form an irreducible representation of an $\mathrm{SU}(2)$ algebra, which can be regarded as the spectrum generating algebra [9] of the half-filled Kondo lattice model. Within the framework of a mean field theory, the magnetic interactions and the Kondo screening are considered on an equal footing, and the ground-state phase diagram of the model Hamiltonian is calculated.

The symmetric Kondo lattice model with anisotropic exchange couplings is defined as:

$$
\begin{aligned}
H & =\sum_{\mathbf{k} \sigma} \epsilon_{\mathbf{k}} c_{\mathbf{k} \sigma}^{\dagger} c_{\mathbf{k} \sigma}+H_{\|}+H_{\perp} \\
H_{\|} & =\frac{J_{\|}}{4} \sum_{i}\left(d_{i \uparrow}^{\dagger} d_{i \uparrow}-d_{i \downarrow}^{\dagger} d_{i \downarrow}\right)\left(c_{i \uparrow}^{\dagger} c_{i \uparrow}-c_{i \downarrow}^{\dagger} c_{i \downarrow}\right) \\
H_{\perp} & =\frac{J_{\perp}}{2} \sum_{i}\left(d_{i \downarrow}^{\dagger} d_{i \uparrow} c_{i \uparrow}^{\dagger} c_{i \downarrow}+d_{i \uparrow}^{\dagger} d_{i \downarrow} c_{i \downarrow}^{\dagger} c_{i \uparrow}\right),
\end{aligned}
$$

where the pseudo-fermion representation for the localized spins $S_{i}^{z}=\left(d_{i \uparrow}^{\dagger} d_{i \uparrow}-d_{i \downarrow}^{\dagger} d_{i \downarrow}\right) / 2, S_{i}^{-}=d_{i \downarrow}^{\dagger} d_{i \uparrow}, S_{i}^{+}=d_{i \uparrow}^{\dagger} d_{i \downarrow}$, has been used with a local constraint $d_{i \uparrow}^{\dagger} d_{i \uparrow}+d_{i \downarrow}^{\dagger} d_{i \downarrow}=1$. Note that $H_{\perp}$ can also be rewritten in the form of

$$
\frac{-J_{\perp}}{4} \sum_{i}\left[\left(d_{i \downarrow}^{\dagger} c_{i \downarrow}+c_{i \uparrow}^{\dagger} d_{i \uparrow}\right)^{2}+\left(d_{i \uparrow}^{\dagger} c_{i \uparrow}+c_{i \downarrow}^{\dagger} d_{i \downarrow}\right)^{2}\right] .
$$

$H_{\|}$describes the polarization of conduction electrons by the local impurity spins, leading to a magnetic instability, while $H_{\perp}$ corresponds to the spin-flip scatterings, giving rise to the local Kondo screening effect. The latter effect has been investigated by various approaches, in particular, those based on a $1 / \mathrm{N}$ expansion [10] ( $\mathrm{N}$ is the degeneracy of the localized spin). However, the former effect is not treated on an equal footing in these approaches because the magnetic interaction occurs there only at $1 / \mathrm{N}^{2}$ order [11].

It has been known that the spectrum generating algebra plays an important role in analyzing complete spectra for a model. For instance, it has been used to study collective excitations and phase transitions in onedimensional metals and two-dimensional Hubbard model 
[9]. Here the spectrum generating algebra for the halffilled Kondo lattice model is given by generators of an $\mathrm{SU}(2)$ Lie group:

$$
\begin{aligned}
\tau^{+} & =\sum_{i} e^{i \mathbf{Q} \cdot \mathbf{r}_{i}}\left(c_{i \uparrow}^{\dagger} d_{i \uparrow}+d_{i \downarrow}^{\dagger} c_{i \downarrow}\right), \\
\tau^{-} & =\sum_{i} e^{-i \mathbf{Q} \cdot \mathbf{r}_{i}}\left(d_{i \uparrow}^{\dagger} c_{i \uparrow}+c_{i \downarrow}^{\dagger} d_{i \downarrow}\right), \\
\tau^{z} & =\frac{1}{2} \sum_{i}\left[\left(c_{i \uparrow}^{\dagger} c_{i \uparrow}-c_{i \downarrow}^{\dagger} c_{i \downarrow}\right)-\left(d_{i \uparrow}^{\dagger} d_{i \uparrow}-d_{i \downarrow}^{\dagger} d_{i \downarrow}\right)\right],
\end{aligned}
$$

which satisfy the commutation relations: $\left[\tau^{z}, \tau^{ \pm}\right]= \pm \tau^{ \pm}$, $\left[\tau^{+}, \tau^{-}\right]=2 \tau^{z}$, the $\mathrm{SU}(2)$ algebra. $\mathbf{Q}$ is the AF reciprocal vector. We also find that an irreducible representation of this $\mathrm{SU}(2)$ algebra can serve as the order parameter operators for this model. They are given by:

$$
\begin{aligned}
K^{+} & =\sum_{i}\left(c_{i \uparrow}^{\dagger} d_{i \uparrow}+d_{i \downarrow}^{\dagger} c_{i \downarrow}\right), \\
K^{-} & =-\sum_{i}\left(d_{i \uparrow}^{\dagger} c_{i \uparrow}+c_{i \downarrow}^{\dagger} d_{i \downarrow}\right), \\
M^{z} & =\frac{1}{2} \sum_{i} e^{i \mathbf{Q} \cdot \mathbf{r}_{i}}\left[\left(d_{i \uparrow}^{\dagger} d_{i \uparrow}-d_{i \downarrow}^{\dagger} d_{i \downarrow}\right)-\left(c_{i \uparrow}^{\dagger} c_{i \uparrow}-c_{i \downarrow}^{\dagger} c_{i \downarrow}\right)\right],
\end{aligned}
$$

with the following commutation relations $\left[\tau^{ \pm}, K^{\mp}\right]=$ $2 M^{z},\left[\tau^{ \pm}, M^{z}\right]=K^{ \pm},\left[\tau^{z}, K^{ \pm}\right]= \pm K^{ \pm}$, and $\left[\tau^{z}, M^{z}\right]=$ 0 , where $K^{ \pm}$are the order parameter operators describing the local Kondo singlet state and $M^{z}$ is the staggered magnetization operator describing the AFLRO in terms of a commensurate spin-density wave. The expectation values of these order operators are the corresponding order parameters. Note that the model Hamiltonian does not satisfy this SU(2) symmetry defined by the spectrum generating algebra, which can however provide us with a guide to choose the order parameters in the model Hamiltonian.

Now we introduce a mean-field theory treating equally the magnetic interactions between the localized spins and the itinerant electron screening aspects. The longitudinal interaction term is approximated as:

$$
\begin{aligned}
& \frac{J_{\|}}{2}\left[m_{d} \sum_{i} e^{i \mathbf{Q} \cdot \mathbf{r}_{i}}\left(c_{i \uparrow}^{\dagger} c_{i \uparrow}-c_{i \downarrow}^{\dagger} c_{i \downarrow}\right)\right. \\
& \left.\quad-m_{c} \sum_{i} e^{i \mathbf{Q} \cdot \mathbf{r}_{i}}\left(d_{i \uparrow}^{\dagger} d_{i \uparrow}-d_{i \downarrow}^{\dagger} d_{i \downarrow}\right)\right]+J_{\|} m_{c} m_{d} \mathcal{N},
\end{aligned}
$$

where the staggered magnetizations have been introduced as the AF order parameters $m_{c}=-\frac{1}{2}<c_{i \uparrow}^{\dagger} c_{i \uparrow}-$ $c_{i \downarrow}^{\dagger} c_{i \downarrow}>e^{-i \mathbf{Q} \cdot \mathbf{r}_{i}}$ and $m_{d}=\frac{1}{2}<d_{i \uparrow}^{\dagger} d_{i \uparrow}-d_{i \downarrow}^{\dagger} d_{i \downarrow}>e^{-i \mathbf{Q} \cdot \mathbf{r}_{i}}$, and a minus sign has been absorbed into the definition of $m_{c}$ for convenience. The transverse interaction term is approximated to be:

$$
\frac{J_{\perp} V}{2} \sum_{i \sigma}\left(c_{i \sigma}^{\dagger} d_{i \sigma}+d_{i \sigma}^{\dagger} c_{i \sigma}\right)+\frac{J_{\perp}}{2} V^{2} \mathcal{N},
$$

with the hybridization order parameter describing the local Kondo singlet state $-V=<c_{i \uparrow}^{\dagger} d_{i \uparrow}+d_{i \downarrow}^{\dagger} c_{i \downarrow}>$ $=<d_{i \uparrow}^{\dagger} c_{i \uparrow}+c_{i \downarrow}^{\dagger} d_{i \downarrow}>$. Moreover, we could also introduce a $d$-electron chemical potential term $\Sigma_{i, \sigma} E_{d} d_{i \sigma}^{\dagger} d_{i \sigma}$ to fix the $d$-electron density to be one on each site, but actually this is not necessary at half filling because the electron-hole symmetry automatically imposes $E_{d}=0$ [2]. Therefore, the mean field Hamiltonian can be written in the following form

$$
H=\sum_{\mathbf{k} \sigma}^{\prime} \Psi_{\mathbf{k} \sigma}^{\dagger} \widehat{H}_{\mathbf{k} \sigma} \Psi_{\mathbf{k} \sigma}+\mathcal{N}\left(J_{\|} m_{c} m_{d}+\frac{J_{\perp} V^{2}}{2}\right),
$$

where $\Psi_{\mathbf{k} \sigma}^{\dagger}=\left(c_{\mathbf{k} \sigma}^{\dagger}, c_{\mathbf{k}+\mathbf{Q} \sigma}^{\dagger}, d_{\mathbf{k} \sigma}^{\dagger}, d_{\mathbf{k}+\mathbf{Q} \sigma}^{\dagger}\right)$, its transposition $\Psi_{\mathbf{k} \sigma}$, and the matrix

$$
\widehat{H}_{\mathbf{k} \sigma}=\left[\begin{array}{llll}
\epsilon_{\mathbf{k}}, & \frac{J_{\|}}{2} m_{d} \sigma, & \frac{J_{\perp} V}{2}, & 0 \\
\frac{J_{\|}}{2} m_{d} \sigma, & -\epsilon_{\mathbf{k}}, & 0, & \frac{J_{\perp} V}{2} \\
\frac{J_{\perp} V}{2}, & 0, & 0, & -\frac{J_{\|}}{2} m_{c} \sigma \\
0, & \frac{J_{\perp} V}{2}, & -\frac{J_{\|}}{2} m_{c} \sigma, & 0
\end{array}\right] .
$$

The static staggered magnetizations partially break the translational symmetry and the Brillouin zone is folded in half, so that the summation over $\mathbf{k}$ is taken in the reduced Brillouin zone. The quasiparticle bands are determined by the equation: $|E \widehat{I}-\widehat{H}|=0$, giving rise to four bands with dispersions,

$$
E_{ \pm \pm}(\mathbf{k})= \pm \frac{1}{\sqrt{2}} \sqrt{\epsilon_{\mathbf{k}}^{2}+J_{\|}^{2}\left(m_{c}^{2}+m_{d}^{2}\right) / 4+J_{\perp}^{2} V^{2} / 2 \pm E^{\prime}(\mathbf{k})}
$$

with

$$
\begin{aligned}
E^{\prime}(\mathbf{k})= & \left\{\left[\epsilon_{\mathbf{k}}^{2}+J_{\|}^{2}\left(m_{c}^{2}+m_{d}^{2}\right) / 4+J_{\perp}^{2} V^{2} / 2\right]^{2}\right. \\
& \left.-\frac{1}{4}\left(J_{\|}^{2} m_{c} m_{d}+J_{\perp}^{2} V^{2}\right)^{2}-J_{\|}^{2} m_{c}^{2} \epsilon_{\mathbf{k}}^{2}\right\}^{1 / 2},
\end{aligned}
$$

and the ground-state energy is given by

$$
E_{g}=2 \sum_{\mathbf{k}}^{\prime}\left[E_{--}(\mathbf{k})+E_{-+}(\mathbf{k})\right]+\mathcal{N}\left(J_{\|} m_{c} m_{d}+\frac{J_{\perp} V^{2}}{2}\right),
$$

corresponding to completely filling the two negative energy bands with electrons. The self-consistent equations are obtained by minimizing the ground-state energy $E_{g}$ with respect to $m_{d}, m_{c}$, and $V$, respectively. After the summations over momenta are transformed into integrals over energies by assuming a constant density of states of the conduction electrons in $[-D, D]$, the self-consistent equations are expressed in the form

$$
\begin{aligned}
& \frac{\lambda_{\|}}{8} \int_{-1}^{1} d \varepsilon \frac{2 F_{2}(\varepsilon) m_{d}+\left(\lambda_{\|}^{2} m_{c} m_{d}+\lambda_{\perp}^{2} V^{2}\right) m_{c}}{2 F_{1}(\varepsilon) F_{2}(\varepsilon)}=m_{c}, \\
& \frac{\lambda_{\|}}{8} \int_{-1}^{1} d \varepsilon \frac{2\left(F_{2}(\varepsilon)+2 \varepsilon^{2}\right) m_{c}+\left(\lambda_{\|}^{2} m_{c} m_{d}+\lambda_{\perp}^{2} V^{2}\right) m_{d}}{2 F_{1}(\varepsilon) F_{2}(\varepsilon)}=m_{d}
\end{aligned}
$$




$$
\frac{\lambda_{\perp}}{4} \int_{-1}^{1} d \varepsilon \frac{2 F_{2}(\varepsilon)+\left(\lambda_{\|}^{2} m_{c} m_{d}+\lambda_{\perp}^{2} V^{2}\right)}{2 F_{1}(\varepsilon) F_{2}(\varepsilon)}=1,
$$

where we have used the simplified expressions in terms of $F_{1}$ and $F_{2}$,

$$
\begin{aligned}
& F_{1}(\varepsilon)=\sqrt{\varepsilon^{2}+\lambda_{\|}^{2}\left(m_{c}^{2}+m_{d}^{2}\right) / 4+\lambda_{\perp}^{2} V^{2} / 2+F_{2}(\varepsilon)}, \\
& F_{2}(\varepsilon)=\sqrt{\frac{1}{4}\left(\lambda_{\|}^{2} m_{c} m_{d}+\lambda_{\perp}^{2} V^{2}\right)^{2}+\lambda_{\|}^{2} m_{c}^{2} \varepsilon^{2}}
\end{aligned}
$$

and we have also used the notations: $\lambda_{\|}=J_{\|} / D$, $\lambda_{\perp}=J_{\perp} / D$, where $2 D$ is the bandwidth of the conduction electrons.

When $\lambda_{\|} \gg \lambda_{\perp}$, the polarization effect described by $H_{\|}$dominates, and the ground state has an AFLRO characterized by finite staggered magnetizations for both localized spins and the conduction electrons. Thus, the total staggered magnetization of the system should be given by $M^{z} / \mathcal{N}=\left(m_{d}+m_{c}\right)$, while the staggered magnetic moment is $\mu=\left(m_{d}-m_{c}\right)$. Let us assume for the moment they are the only order parameters, i.e. $V=0$. All equations involved are greatly simplified. Due to unit cell doubling the conduction electron band is folded in half to form two new bands: $\epsilon_{ \pm}(\mathbf{k})= \pm \sqrt{\epsilon_{\mathbf{k}}^{2}+\left(J_{\|} m_{d}\right)^{2} / 4}$. There is a gap in the conduction electron excitation spectrum: $\Delta=J_{\|} m_{d} / 2$. The charge gap is twice this value, $\Delta_{c h}=J_{\|} m_{d}$. Moreover, the ground-state energy of the AFLRO state can also be found

$$
\frac{E_{g}^{A F}}{\mathcal{N} D}=-\frac{1}{2}\left[\sqrt{1+\left(\frac{\lambda_{\|}}{4}\right)^{2}}+\left(\frac{\lambda_{\|}}{4}\right) \frac{m_{c}}{m_{d}}\right],
$$

where $\mathcal{N}$ is the total number of the lattice sites, and $\frac{m_{c}}{m_{d}}=\frac{\lambda_{\|}}{4} \ln \left(\frac{4}{\lambda_{\|}}+\sqrt{1+\left(\frac{4}{\lambda_{\|}}\right)^{2}}\right)$ with $m_{d}=\frac{1}{2}$.

In the opposite limit, when $\lambda_{\perp} \gg \lambda_{\|}$, the Kondo screening effect described by $H_{\perp}$ dominates, and the ground state has a finite local Kondo order parameter $V$. If we assume again for the moment it is the only order parameter, the system is a nonmagnetic band insulator with an effective hybridization: $\frac{1}{V}=\lambda_{\perp} \sinh \left(\frac{1}{\lambda_{\perp}}\right)$. A similar result has already been given by the $1 / \mathrm{N}$ expansion approach [10], which is believed to describe the correct low-energy Kondo physics. The quasiparticle excitation spectrum is expressed in the form $\epsilon_{ \pm}^{\prime}(\mathbf{k})=$ $\frac{1}{2}\left(\epsilon_{\mathbf{k}} \pm \sqrt{\epsilon_{\mathbf{k}}^{2}+\left(J_{\perp} V\right)^{2}}\right)$, and there is a small hybridization gap $\Delta_{h y} \simeq\left(J_{\perp} V\right)^{2} / 2 D$, which splits the Kondo resonance formed at the Fermi level. At half filling, each lattice site has one conduction electron and one localized spin, and they can form a local singlet state, thus the system becomes a disordered nonmagnetic insulator, a band-insulator with both charge and spin gap $2 \Delta_{h y}$. The ground-state energy can be also calculated

$$
\frac{E_{g}^{K S}}{\mathcal{N} D}=-\frac{1}{2} \operatorname{coth}\left(\frac{1}{\lambda_{\perp}}\right)
$$

Physically, this disordered Kondo singlet state is adiabatically connected to the usual Kondo spin liquid state [2].

Comparing the respective ground-state energies $E_{g}^{K S}$ and $E_{g}^{A F}$, we find the phase boundary between the two ground states:

$$
\begin{aligned}
\left(\frac{1}{\lambda_{\perp}}\right)_{c}=\operatorname{coth}^{-1} & {\left[\sqrt{1+\left(\frac{\lambda_{\|}}{4}\right)^{2}}\right.} \\
& \left.+\left(\frac{\lambda_{\|}}{4}\right)^{2} \ln \left(\frac{4}{\lambda_{\|}}+\sqrt{1+\left(\frac{4}{\lambda_{\|}}\right)^{2}}\right)\right],
\end{aligned}
$$

which is displayed by the solid line in Fig.1. Below this line, the AFLRO state is more stable $E_{g}^{A F}<E_{g}^{K S}$; above this line the disordered Kondo singlet state is more stable. We note that this line intersects the diagonal $\lambda_{\perp}=\lambda_{\|}$ at 0.58 . Taking into account that the cut-off parameter $D=2 t$ with $t$ as the nearest neighbor hopping, in two dimensions, where our assumption of a constant density of states is better justified, we find $J_{c} / t=1.16$. This value is not far from the numerical results [4] $J_{c} / t=$ $1.40 \sim 1.45$, obtained for the isotropic model.

Now we turn to discuss the main new result of this paper, namely the possible coexistence of AFLRO with Kondo singlet state. In fact, our system of self-consistent equations allows solutions with all order parameters $m_{d}, m_{c}$ and $V$ being nonzero. Let's start from the region where $\mathrm{AF}$ order dominates, with finite $m_{d}$ and $m_{c}$ to see where the instability towards the Kondo singlet state emerges. Assume $V$ being small, but non-zero, we obtain the following solution,

$$
\begin{aligned}
& \left(\frac{1}{\lambda_{\perp}}\right)_{c 2}=\frac{2}{\lambda_{\|}} \frac{m_{c}}{m_{d}} \\
& \quad+\sqrt{\frac{1-\frac{m_{c}}{m_{d}}}{1+\frac{m_{c}}{m_{d}}}} \tan ^{-1}\left(\sqrt{\frac{1-\frac{m_{c}}{m_{d}}}{1+\frac{m_{c}}{m_{d}}}} \tanh \left(\frac{m_{c}}{m_{d}} \frac{2}{\lambda_{\|}}\right)\right),
\end{aligned}
$$

where $\frac{m_{c}}{m_{d}}=\frac{\lambda_{\|}}{4} \ln \left(\frac{4}{\lambda_{\|}}+\sqrt{1+\left(\frac{4}{\lambda_{\|}}\right)^{2}}\right)$. This instability line for the AF state is delineated by the thick dotted line in Fig.1. In a similar way, we can also determine the instability boundary in the Kondo singlet phase, which corresponds to the appearance of small AF order parameters $m_{c}$ and $m_{d}$. From the above self-consistent equations, we can derive the critical value of the coupling parameter

$$
\left(\frac{1}{\lambda_{\|}}\right)_{c 1}=\frac{\lambda_{\perp} \sinh \left(\frac{2}{\lambda_{\perp}}\right)-2}{4 \lambda_{\perp}\left(\sqrt{\lambda_{\perp} \sinh \left(\frac{2}{\lambda_{\perp}}\right)-1}-1\right)}
$$

and a relation between $m_{c}$ and $m_{d}: \frac{m_{c}}{m_{d}}=\frac{\lambda_{\|}}{4 \lambda_{\perp}-\lambda_{\|}}$. This instability line for the disordered Kondo singlet state is displayed by a thin dotted line in Fig. 1 . 
Between these two instability lines $\left(\lambda_{\perp c 1}<\lambda_{\perp}<\right.$ $\left.\lambda_{\perp c 2}\right)$, there is a narrow regime where the AF and the local Kondo singlet screening order parameters coexist to balance the energy gain from the transverse and longitudinal exchange couplings. The disordered Kondo singlet state and the AFLRO state can both be used to describe two distinct ground states of the conventional Kondo insulators. It seems to us that a new phase may be present when the exchange coupling parameters are tuned carefully. In such a new phase, the dynamical magnetic structure factor should have two contributions: a qindependent single site slow component, which is typical of the localized Kondo-type excitations, and a strongly q-dependent intersite fast component, reflecting the magnetic interactions. These features can be detected in inelastic neutron scattering experiments for some Kondo insulating materials. Moreover, since the localized spins are partially screened by the conduction electrons, and the residual localized spins still have weak AF long-range correlations mediated by the polarization of the conduction electrons. This novel feature may be related to the small magnitude of the induced staggered magnetic moments for $\mathrm{URu}_{2} \mathrm{Si}_{2}$ and $\mathrm{UPt}_{3}$, the so-called heavy fermion micromagnetism [12].

We realize that our result is based on a mean field calculation, and its validity should be further checked by more rigorous analytic and numerical treatments. Eventually, it should be verified by experiments. We also note that the numerical results for the isotropic model [4,5.7] indicate a second order phase transition between the Kondo singlet and AFLRO states. However, the order of phase transitions is a very sensitive issue, and the isotropic case may well be a degenerate point of the more general anisotropic model we consider here.

In conclusion, we have considered a half-filled anisotropic Kondo lattice model within a mean field theory. The ground-state phase diagram has been calculated. In addition to the AFLRO phase and the Kondo singlet phase, we have found that both of these two distinct phases can coexist as a result of the balance between the Kondo screening effects and the magnetic interactions, which provides a possible new ground state for the Kondo insulating compounds.

\section{Acknowledgments}

One of the authors (G. -M. Zhang) wishes to thank ZhiLiang Cao, Qiang Gu, and Xiao-Bin Wang for their useful discussions and help, and would also like to express his gratitude to International Center for Theoretical Physics (Trieste, Italy) for the hospitality, where this work was initiated.

[1] S. Doniach, Physica B 91, 231 (1977).
[2] H. Tsunetsugu, M. Sigrist, and K. Ueda, Rev. Mod. Phys. 69, 809 (1997), and references therein.

[3] C. Lacroix and M. Cyrot, Phys. Rev. B 20, 1969 (1979).

[4] Z. Wang, X. P. Li, and D. H. Lee, Physica B 199-200, 463 (1984).

[5] Z. P. Shi, R. R. P. Singh, M. P. Gelfand, Z. Wang, Phys. Rev. B 51, 15630 (1995).

[6] M. Vekic, J. W. Cannon, D. J. Scalapino, R. T. Scaletta, and R. L. Suger, Phys. Rev. Lett. 74, 2367 (1995).

[7] F. F. Assaad, Phys. Rev. Lett. 83, 796 (1999).

[8] M Jarrel, Phys. Rev. B 51, 7429 (1995).

[9] A. I. Solomon and J. L. Birman, J. Math. Phys. 28, 1526 (1987); A. B. Eriksson, T. Einarsson, and S. Ostlund, Phys. Rev. B 52, 3662 (1995); R. S. Markiewicz and M. T. Vaughn, cond-mat/9802078.

[10] N. Read and D. N. Newns, J. Phys. C 16, 3273 (1983); P. Coleman, Phys. Rev. B 29, 3035 (1984).

[11] S. Doniach, Phys. Rev. B 35, 1814 (1987).

[12] G. Aeppli, E. Bucher, C. Broholm, J. K. Kjems, J. Baumann, and J. Hufnagl, Phys. Rev. Lett. 60, 615 (1988); E. D. Isaacs, D. B. McWhan, R. N. Kleiman, D. J. Bishop, C. E. Ice, P. Zschack, B. D. Gaulin, T. E. Mason, J. D. Garrett, and W. J. L. Buyers, ibid. 65, 3185 (1990).

Figure Caption

Fig.1. The ground-state phase diagram of the halffilled anisotropic Kondo lattice model in the $\lambda_{\perp}-\lambda_{\|}$ plane. The narrow area between the lines of $\left(\lambda_{\perp}\right)_{c 1}$ and $\left(\lambda_{\perp}\right)_{c 2}$ is the regime where the local Kondo singlet state and the AFLRO may coexist. The solid line corresponds to the boundary of $E_{g}^{A F}=E_{g}^{K S}$, and the thin dashed line denotes the isotropic limit of the model. 
Figure 1

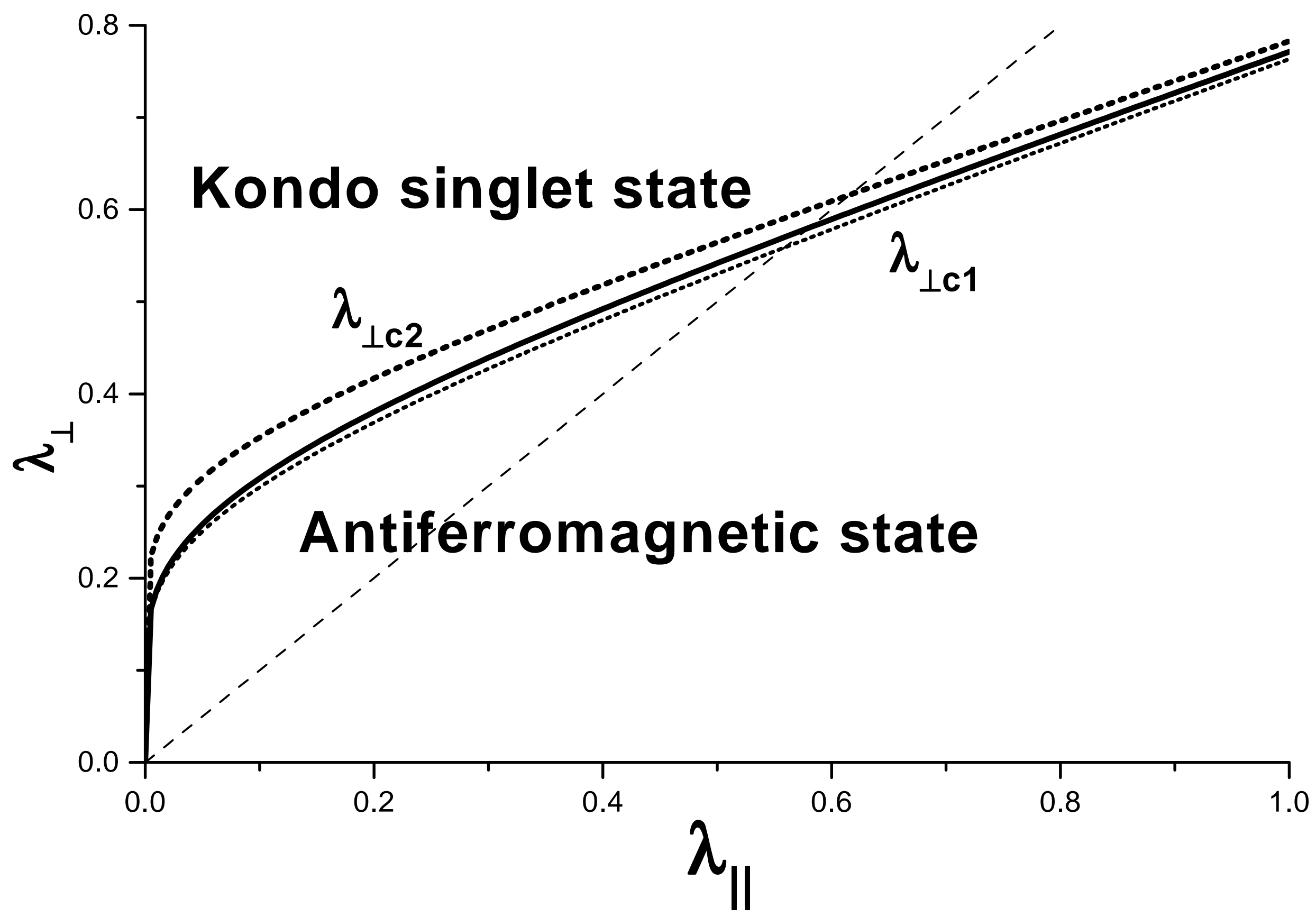

